\title{
Graphene antidot lattices as potential electrode materials for
}

\author{
supercapacitors \\ Lizhao Liu ${ }^{1,2}$, Xin Yue ${ }^{3}$, Jijun Zhao ${ }^{2 *}$, Qian Cheng ${ }^{4}$, Jie Tang ${ }^{4}$ \\ ${ }^{1}$ School of Science, Dalian University of Technology, Panjin Campus, Panjin 124221, China \\ ${ }^{2}$ Key Laboratory of Materials Modification by Laser, Ion and Electron Beams (Dalian University of Technology), Ministry of \\ Education, Dalian 116024, China \\ ${ }^{3}$ Yangzhou Institute of Energy and Material, Chinese Academy of Sciences, Yangzhou 225127, China \\ ${ }^{4}$ National Institute for Materials Science, 1-2-1 Sengen, Tsukuba, Ibaraki, 305-0047, Japan
}

\section{Abstract}

Thermodynamic stabilities and electronic properties of graphene antidot lattices with hexagonal holes were examined using density functional theory calculations and several crucial factors related to the applications of supercapacitors were discussed. For the graphene antidot lattices with different hole sizes, the formation energy per edge length is about $0.50 \sim 0.60 \mathrm{eV} / \mathrm{nm}$, which is comparable to that of graphene nanoribbon edges. Within a hole density of $10 \%$, the graphene antidot lattices can maintain the excellent electronic properties of perfect graphene due to negligible intervalley scattering. Further increasing the hole density will open a band gap. Taking the potassium chloride ( $\mathrm{KCl}$ ) electrolyte as an example, we further investigated the diffusion behaviors of potassium $(\mathrm{K})$ and chlorine $(\mathrm{Cl})$ atoms through the graphene antidot lattices. It was shown that $\mathrm{K}$ and $\mathrm{Cl}$ atoms can go through the holes with nearly no barrier at an appropriate hole size of $0.54 \mathrm{~nm}$, which gives an optimum pore diameter of $\sim 0.86 \mathrm{~nm}$. Therefore, the excellent graphene-like electronic properties and

\footnotetext{
* Corresponding author. Tel./Fax: +86 41184706100. E-mail address: zhaoji@dlut.edu.cn (J. Zhao).
} 
good penetrability for ions suggest promising applications of graphene antidot lattices in the field of supercapacitors.

Keywords: Graphene antidot lattices, Supercapacitors, Stability, Density of states, Diffusion

\section{Introduction}

Supercapacitors, also called electric double layer capacitors (EDLCs), have attracted great attentions due to their advantages of high power density and reliability, low maintenance and weight, fast charge and release propagation, and long cycle life [1, 2]. Generally, specific capacitance $\left(\mathrm{F} \mathrm{g}^{-1}\right)$ of one supercapacitor follows the rule of a parallel-plate capacitor, where the capacitance is proportional to surface area of the electrodes [3]. Therefore, searching for appropriate electrode materials is of key importance for improving the performance of supercapacitors. Synthesis of graphene [4] greatly promotes the R\&D of EDLCs due to its large specific surface area up to $2630 \mathrm{~m}^{2} \mathrm{~g}^{-1}$ [5] and superior electric conductivity [6, 7]. Nowadays, the EDLCs with graphene-based electrodes have been extensively explored and achieved high performance [3, 8-11]. Especially, the theoretical value of $550 \mathrm{~F} \mathrm{~g}^{-1}$ calculated for single-layer graphene [1] was almost achieved by using composites of graphene layers with polymer matrix as electrodes [12].

Although a high theoretical value of specific capacitance was predicted, it is hard to achieve such a value in real samples. Specif ic capacitances of $130 \mathrm{~F} \mathrm{~g}^{-1}$ in aque ous potassium hydroxide and $99 \mathrm{~F} \mathrm{~g}^{-1}$ in an organic electrolyte were reported in the graphene-based EDLCs [1], which were far below the theoretical value. There are, therefore, some efforts in improving the performance of supercapacitors by modification of graphene electrodes. Promisingly, supercapacitors with porous graphene can greatly enhance the specific 
capacitance. It was reported that the EDLCs with porous graphene sheets as the electrodes exhibit a specific capacitance of $154 \mathrm{~F} \mathrm{~g}^{-1}$ at $500 \mathrm{mV} \mathrm{s}^{-1}$ in $6 \mathrm{M} \mathrm{KOH}$ compared to $67 \mathrm{~F} \mathrm{~g}^{-1}$ for perfect graphene sheets [13]. Supercapacitors produced by active graphene with primarily 0.6- to 5-namometer-width pores can achieve a specific capacitance of $200 \mathrm{~F} \mathrm{~g}^{-1}$ at a current density of $0.7 \mathrm{~A} \mathrm{~g}^{-1}$ [14]. More excitingly, graphene nanomesh [15], a kind of porous graphene with periodic distribution of uniform sized holes, was found as a promising candidate for supercapacitor electrodes with a specific capacitance up to $255 \mathrm{~F} \mathrm{~g}^{-1}$, long cycle life of 2000 cycles and excellent rate performance [16].

Previously, it was found that graphene sheet is impermeable even for helium atom [17]. Incorporation of holes on graphene electrode can thus act as doors for electrolyte ions to pass through and enhance the diffusivity, which can both improve the specific capacitance and shorten the charge-release time $[18,19]$. Meanwhile, existence of holes on the graphene sheet will demolish the thermodynamic stability and reduce the electric conductivity of the graphene electrode; both effects are harmful for the supercapacitor applications. Hence, there must be some compromise to enhance performance of the EDLCs with appropriate incorporation of holes in the graphene electrodes, which deserves a comprehensive investigation at atomistic scale. Considering of above factors, graphene antidot lattice [20], a typical graphene-based structure with periodic distribution of holes and tunable electronic properties, may be a suitable candidate for the electrode materials [21]. It was reported that the electronic properties of graphene antidot lattices vary with hole shape and even opening of band gap and induced magnetism can be obtained by carefully controlling the holes [22, 23]. Among different shaped holes, graphene antidot lattices with hexagonal holes usually possess 
better thermodynamic stability than the others [24]. Also, edge reconstruction usually formed the hexagon-like holes experimentally [25]. In particular, regularly hexagonal holes on graphene have been recently fabricated by surface-assisted coupling of specifically designed molecular building blocks [26] or by electron beam lithography [27]. Therefore, here we focus on the graphene antidot lattices with hexagonal holes.

Previous theoretical studies on the graphene antidot lattices focused on the ir geometries and electronic properties [20, 22-24], whereas little is known about the ir performance as electrode materials for the EDLCs from the theoretical point of view. In this paper, using graphene antidot lattices with hexagonal holes as representatives, we exploited several critical issues for their technological applications in the field of supercapacitors. We found that thermodynamic stabilities and electron density of states (DOS) of the graphene antidot lattices are closely related to the geometric parameters of the hexagonal holes, including hole density $(R)$ and hole size $(r)$. With low hole density $(R<10 \%)$, graphene antidot lattices can preserve the excellent electronic properties of the pristine graphene. Moreover, the existence of hole facilitates the diffusion of ions through the graphene sheet. These theoretical results provide some useful clues to tailor the thermodynamic stabilities and electronic properties of the graphene antidot lattices and shed some lights on their potential applications as electrode materials for the EDLCs.

\section{Structural models}

Starting from a perfect graphene monolayer, we introduce a series of equilateral hexagonal holes to construct structural models of graphene antidot lattices. All dangling bonds on the periphery of holes are passivated by $\mathrm{H}$ atoms since the graphene-based electrode 
materials directly interact with the electrolyte in real EDLCs, where the acid aqueous solution contains $\mathrm{H}^{+}$ions [12]. Here hole density $R$ is defined by the following equation:

$$
R=S_{h} / S_{c}
$$

where $S_{h}$ and $S_{c}$ are the areas of a hexagonal hole and the whole supercell, respectively. If we label the side length of a hole as $l$, as shown in Fig. 1(a), $R$ becomes,

$$
R=\frac{3 \sqrt{3}}{2} \frac{l^{2}}{a b}
$$

where $a$ and $b$ are lattice parameters of the supercell in the $x$ and $y$ directions, respectively. Meanwhile, the hole size $r$ can be also related to $l$ by $r=\sqrt{3} l / 2$.

Fixing the parameter $r$, structural models of different hole densities can be obtained by varying the size of the supercell, i.e., enlarging the parameters $a$ and $b$. Here, we increase $a$ and $b$ evenly via keeping a nearly constant $a / b$ ratio of $\sim 1.00$. Fig. 1(a) presents representative models for the graphene antidot lattices with the minimum size for different $R$ 's. The number of carbon and hydrogen atoms in the supercell varies from $\mathrm{C}_{26} \mathrm{H}_{6}$ for $R=43.9 \%$ to $\mathrm{C}_{282} \mathrm{H}_{6}$ for $R=4.9 \%$.

In addition, structural models of different hole sizes are also constructed. As shown in Fig. 1(b), four structural models with $r=0.33,0.54,0.75$, and $0.96 \mathrm{~nm}$ were created. For all these four structural models, the closest distance between two neighboring holes (side-side distance) is $0.14 \mathrm{~nm}$ along the $a$ direction and $0.25 \mathrm{~nm}$ along the $b$ direction. Note that the choice of high hole density ( $\sim 50 \%)$ or short hole-hole distance is to minimize the computational cost due to the large supercells (up to 104 carbon atoms and 24 hydrogen atoms), while the effect of hole size obtained from the present models would still be valid for the other situations of hole densities. 


\section{Computational me thods}

Periodic first-principles computations were performed using the plane-wave pseudopotential technique implemented in the PWSCF code based on the density functional theory (DFT) [28]. The Perdew-Burke-Ernzerhof (PBE) functional in the generalized gradient approximation (GGA) was employed to describe the exchange-correlation energy [29]. The ultrasoft pseudopotential [30] was adopted for describing the ion-electron interactions. A well-tested kinetic energy cutoff of 30 Ry for the plane-wave basis set was used in geometric optimizations and electronic structure calculations of the graphene antidot lattices. To simulate the diffusion behaviors, a higher kinetic energy cutoff of 55 Ry was adopted to ensure well convergence of the total energies due to incorporation of $\mathrm{K}$ and $\mathrm{Cl}$ atoms. The Monkhorst-Pack k-point grids [31] with a separation of $0.03 \AA^{-1}$ were chosen for the total energies and $0.01 \AA^{-1}$ for DOS calculations. The thickness of the vacuum layer perpendicular to the graphene plane was chosen as $15 \AA$ to avoid the interaction between the graphene sheet and its periodic images. The Brillouin zone integrations were performed using the Gaussian-spreading special-point technique [32] with a smearing parameter of 0.005 Ry. During geometry optimization, the size of the supercell dimensions ( $a$ and $b$ ) were relaxed but the rectangular shape of supercell was retained. For all calculations, the convergence criteria of less than $10^{-7}$ Ry/atom with respect to the total energy and less than $0.001 \mathrm{Ry} / \AA$ with regard to force on atom were adopted.

To simulate the diffusion behaviors of $\mathrm{K}$ and $\mathrm{Cl}$ atoms, a rigid lattice model was adopted for $\mathrm{K} / \mathrm{Cl}$ penetrating the hole without varying the supercell parameters or considering the temperature. At the beginning, we optimized the system to find out the optimal distance $\left(d_{o}\right)$ 
to place the $\mathrm{K} / \mathrm{Cl}$ atom on the graphene antidote lattices, as shown in Fig. 2. Then, the $\mathrm{K} / \mathrm{Cl}$ atom was moved towards the hole and reached a maximum diffusion barrier until arriving at basal planes of the graphene antidote lattices. For reference, the diffusion behaviors of $\mathrm{K}$ and $\mathrm{Cl}$ atoms going through the perfect graphene monolayer were also studied.

\section{Results and discussion}

\subsection{Formation energies of the grapheme antidot lattices}

We first considered the dependence of formation energy on the hole size. For a sufficiently large hole, its formation energy mainly originates from creation of the hole periphery. Then, the formation energy should be proportional to the edge length $(L)$ of the hole. Therefore, we defined the formation energy per edge length to describe the thermodynamic stability, which is formulated as

$$
E_{\text {form }}(L)=\left(E_{C_{m} H_{n}}-m E_{C}-\frac{n}{2} E_{H_{2}}\right) / L,
$$

where $E_{C_{m} H_{n}}, E_{C}$ and $E_{H_{2}}$ are the total energies of the graphene antidot lattice, the perfect graphene per atom and an isolated $\mathrm{H}_{2}$ molecule, respectively. Meanwhile, $L=6 l$ is the edge length of the hexagonal hole.

For the graphene antidot lattices shown in Fig. 1(b), calculated $E_{f o r m}(L)$ is summarized in table 1 . One can see that the formation energy ranges between $0.53 \mathrm{eV} / \mathrm{nm}$ and $0.57 \mathrm{eV} / \mathrm{nm}$. With increasing hole size, it gradually approach about $0.60 \mathrm{eV} / \mathrm{nm}$ with slight fluctuation. Intuitively, as the hole size tends to infinity, the formation energy per edge length should be equivalent to the formation energy for hydrogen terminated zigzag graphene nanoribbon, which is also $0.60 \mathrm{eV} / \mathrm{nm}$ from a previous DFT calculation [33]. 
In addition to the hole size effect, the influence of hole density on the thermodynamic stabilities of the graphene antidot lattices was also investigated by examining the formation energies of the holes with various hole density as indicated in Fig. 1(a). Fig. 3 displays $E_{\text {form }}$ $(L)$ as a function of $R$. As mentioned above, the formation energy should be proportional to be total edge length of the hole. Since the hole size $(r \sim 0.33 \mathrm{~nm})$ and total edge length $(L \sim 2.28$ $\mathrm{nm}$ ) are nearly the same for structures with different hole densities, the formation energy per edge length remains invariant as $0.54 \pm 0.01 \mathrm{eV} / \mathrm{nm}$.

In brief, analys is of the formation energy suggest that creating zigzag-edged holes of different sizes or densities on graphene sheet only costs moderate energy $(0.50 \sim 0.60 \mathrm{eV} / \mathrm{nm})$, which is comparable to that of the zigzag edge of graphene. Indeed, this kind of hexagonal holes on graphene layer has been successfully fabricated in experiments [26, 27]. The hole radius reported in Ref. [26] was about $0.37 \mathrm{~nm}$ with a pore spacing of $0.74 \mathrm{~nm}$, which are comparable to the present structural models. Meanwhile, much larger holes $(75 \mathrm{~nm}$ in radius with a pore spacing of $20 \mathrm{~nm}$ and $150 \mathrm{~nm}$ in radius with a pore spacing of $40 \mathrm{~nm}$ ) were reported in Ref. [27].

\subsection{Electron density of states}

The electron DOS for different graphene antidot lattices were calculated to study the effect of holes on the electronic properties of the graphene. Fig. 4(a) is the calculated electron DOS of the graphene antidot lattices with different $R$ values. Within $R<10 \%$, the existence of holes has only little influence on the DOS of the perfect graphene. Hence, the excellent electric conductivity of pristine graphene is still retained. However, as $R$ becomes larger $(R>$ $12 \%$ ), a distinct band gap is opened due to interaction between the neighboring holes. For 
example, at $R=12.7 \%$, a moderate band gap of $0.25 \mathrm{eV}$ can be observed. Fig. 4(b) presents the relationship between the hole density and band gap corresponding to the DOS in Fig. 4(a). In general, electronic properties of the graphene antidot lattices can be tuned by varying hole dens ity, providing an effective approach to modify the electric conductivity of graphene-based materials.

Our present theoretical results also coincide well with the previous DFT calculations. In the case of low hole densities ( $R<10 \%$ ), part of carriers may pass through the hole via quantum tunneling on account of the long mean free path of carriers in graphene [34, 35]. Thus, the DOS resembles that of the perfect graphene, which is consistent with the results reported by He et al [22]. However, at high hole densities ( $R>12 \%)$, the distance between the holes is shorter than $1 \mathrm{~nm}$ and hole-hole interactions may build up a periodic potential to trap the electrons and open the band gap, which is referred to as the intervalley scattering proposed in literature [36-38].

Fig. 5 depicts the effect of parameter $r$ on the electron DOS of the graphene antidot lattices. For each $r$, there is always a band gap since $R$ is as high as $\sim 50 \%$ for these systems. Band gaps of $0.24,0.65,0.29$ and $0.17 \mathrm{eV}$ were obtained for $r=0.33,0.54,0.75$ and $0.96 \mathrm{~nm}$, respectively. However, there no clear trend on the hole size for either the magnitude of band gap or the shape of DOS, probably due to strong hole-hole interaction in the systems with large $R$.

\subsection{Diffusion behaviors of K and Clatoms}

As mentioned above, charge-release time is another critical issue for the EDLCs in addition to the capacitance $[18,19]$, which is determined by the diffusion rate of ions. 
Considering that it is difficult to add charges on specific atoms in supercells, here we take the neutral $\mathrm{K}$ and $\mathrm{Cl}$ atoms as representatives to simulate the diffusion behaviors of $\mathrm{K}^{+}$and $\mathrm{Cl}^{-}$ ions for penetrating the graphene antidot lattices. We first optimized the structure to find the optimal distance. Starting from optimized distance $d_{o}$, the $\mathrm{K} / \mathrm{Cl}$ atom was moved towards the hole and then penetrated the hole through its center. The maximum diffusion barrier can be obtained by compared the total energy difference between the initial state and the final state where $\mathrm{K} / \mathrm{Cl}$ atom reaches the basal plane of the graphene or graphene antidote lattice.

The $d_{o}$ for $\mathrm{K}$ atom placed on graphene and graphene antidot lattice is $2.66 \AA$ and $1.95 \AA$ for $r$ of $0.33 \mathrm{~nm}, 3.00 \AA$ for $r$ of $0.54 \mathrm{~nm}$ and $4.72 \AA$ for $r$ of $0.75 \mathrm{~nm}$, respectively. On the other hand, The $d_{o}$ for $\mathrm{Cl}$ atom placed on graphene and graphene antidot lattice is $3.23 \AA$ and $1.79 \AA$ for $r$ of $0.33 \mathrm{~nm}, 0.04 \AA$ for $r$ of $0.54 \mathrm{~nm}$ and $0.43 \AA$ for $r$ of $0.75 \mathrm{~nm}$, respectively. Generally, the optimal distance shows no regular tendency as the hole size changes.

Table 2 summarizes the maximum diffusion barrier for the $\mathrm{K}$ and $\mathrm{Cl}$ atoms. It can be noticed that for both the $\mathrm{K}$ and $\mathrm{Cl}$ atoms, the diffusion barrier dramatically reduces with increasing hole size $r$, and then drops to zero as the $r$ large enough. Moreover, it is impermeable for $\mathrm{K}$ and $\mathrm{Cl}$ atoms going through the graphene since the energy barrier is as high as $\sim 50 \mathrm{eV}$, which agrees with previous reports [17]. For $\mathrm{K}$ atom, it needs to overcome an energy barrier up to $73.98 \mathrm{eV}$ to penetrate the graphene layer, which means impermeable. While, when a hole with $r$ of $0.33 \mathrm{~nm}$ was formed, the diffusion barrier sharply drops to 3.52 $\mathrm{eV}$. At a hole size of $0.54 \mathrm{~nm}$, the $\mathrm{K}$ atom can easily penetrate the hole almost without barrier. Further enlarging the hole size scarcely changes the diffusion barrier. Similarly, it is impermeable for $\mathrm{Cl}$ atom to go through the graphene due to a large barrier of $45.23 \mathrm{eV}$. As the 
hole size increases to $0.33 \mathrm{~nm}$, the diffusion barrier dramatically decreases to $2.04 \mathrm{eV}$ and further trends to zero with the hole size enlarging to $0.54 \mathrm{~nm}$. Also, further increasing the hole size shows little effects on the diffusion barrier.

The present results suggest that a hole size of $0.54 \mathrm{~nm}$ is appropriate for the diffusion of both the $\mathrm{K}$ and $\mathrm{Cl}$ atoms through the holes with nearly no energy barriers. Further increasing the hole size hardly favors the penetration of $\mathrm{K} / \mathrm{Cl}$ atom but creates larger holes, which significantly affect the thermodynamic stability and electric properties of the graphene sheet. In other words, the moderate hole with a radius of $0.54 \mathrm{~nm}$ should be the optimal size corresponding to the best performance of graphene-antidot-lattice-based supercapacitors.

Previously, many efforts have been devoted to search the optimal size for the best performance of the electrode materials, which should depend on the ion size and the operating voltage [39]. For carbide-derived carbon materials in a solvent-free electrolyte $\left(\mathrm{EMI}^{+}\right.$and $\mathrm{TFSI}^{-}$) at $60{ }^{\circ} \mathrm{C}$, the maximum capacitance was obtained at a best hole diameter of $\sim 0.7 \mathrm{~nm}$ [40], which was further confirmed by the grand canonical Monte Carlo simulations [41]. Similarly, an optimal hole diameter of $0.7 \sim 0.8 \mathrm{~nm}$ in other organic electrolytes was also obtained [42, 43]. In addition to the organic electrolytes, Raymundo-P iňero et al. pointed out a pore diameter of $\sim 0.7 \mathrm{~nm}$ could achieve the maximum capacitance for aqueous electrolytes, such as $\mathrm{KOH}$ and $\mathrm{H}_{2} \mathrm{SO}_{4}$ electrolytes [42]. Also, Chmiola et al suggested pores with a diameter of $0.8 \sim 1 \mathrm{~nm}$ can lead to the highest specific surface capacitance in $\mathrm{H}_{2} \mathrm{SO}_{4}$ electrolyte [44]. Here we obtained an optimal radius of $0.54 \mathrm{~nm}$ for the $\mathrm{KCl}$ electrolyte, i.e. $1.08 \sim 1.50$ $\mathrm{nm}$ in pore diameter, which is close to the pore diameter of $\sim 0.7 \mathrm{~nm}$ in $\mathrm{KOH}$ electrolyte proposed by Raymundo-P iňero [42]. Note that the hole size defined in our w ork includes an 
extra $\mathrm{C}-\mathrm{H}$ bond length $(0.11 \mathrm{~nm})$. Therefore the actual pore diameter should be around 0.86 $\mathrm{nm}$ after subtracting the $\mathrm{C}-\mathrm{H}$ bond length, which agrees well with pre vious results $(0.7 \sim 1 \mathrm{~nm})$. Moreover, porous graphene with pore diameter of $\sim 0.74 \mathrm{~nm}$ has been successfully achieved in Bieri’s experiments [26].

\section{Conclusions}

In summary, periodic hexagonal holes were introduced into the perfect graphene to construct the structural models of the graphene antidot lattices. The effects of hole size and hole density on the thermodynamic stabilities and electronic properties have been investigated us ing first-principles calculations. Generally, the graphene antidot lattices with different hole sizes and densities have reasonable thermodynamic stabilities with a formation energy per edge length about $0.50-0.60 \mathrm{eV} / \mathrm{nm}$, which is almost as stable as graphene nanoribbon with H-passivated edges. Furthermore, a critical hole dens ity of $\sim 10 \%$ was obtained, below which the graphene antidot lattices can possess excellent electric properties resembling that of pristine graphene. In addition, compared with the impermeable perfect graphene, the graphene antidot lattices possess improved penetrability for the electrolyte ions. The appropriately sized holes $(r \sim 0.54 \mathrm{~nm})$ on the graphene antidot lattices make penetration of the $\mathrm{K}$ and $\mathrm{Cl}$ atoms easily, which suggests an optimal pore diameter of about $0.86 \mathrm{~nm}$. Over speaking, the reasonable thermodynamic stability, excellent electronic properties, and good ion penetrability make the graphene antidot lattices promising electrode materials for the supercapacitors.

\section{Acknowledgements}

We thank Dr. Taizo Sasaki in NIMS for the suggestive discussions. This work is 
supported in China by the National Natural Science Foundation of China (No. 11134005), the Fundamental Research Funds for the Central Universities of China (DUT14RC(3)114), and the Natural Science Foundation of Jiangsu Province (BY2013064, BK20130458, BK20130431, and BK2012255), and in Japan by the JSPS Grants-in-Aid for Scientific Research No. 22310074, JST ALCA Program, and the Nanotechnology Network Project of the Ministry of Education, Culture, Sports, Science and Technology (MEXT). 


\section{References}

[1] M.D. Stoller, S. Park, Y. Zhu, J. An, R.S. Ruoff, Nano Lett. 8 (2008) 3498.

[2] P. Simon,Y. Gogotsi, Nat. Mater. 7 (2008) 845.

[3] L.L. Zhang,X.S. Zhao, Chem. Soc. Rev. 38 (2009) 2520.

[4] K.S. Novoselov, A.K. Geim, S.V. Morozov, D. Jiang, Y. Zhang, S.V. Dubonos, I.V. Grigorieva, A.A. Firsov, Science 306 (2004) 666.

[5] L.L. Zhang, R. Zhou, X.S. Zhao, J. Mater. Chem. 20 (2010) 5983.

[6] Y. Hernandez, V. Nicolosi, M. Lotya, F.M. Blighe, Z. Sun, S. De, I.T. McGovern, B. Holland, M. Byrne, Y.K. Gun'Ko, J.J. Boland, P. Niraj, G Duesberg, S. Krishnamurthy, R. Goodhue, J. Hutchis on, V. Scardaci, A.C. Ferrari, J.N. Coleman, Nat. Nanotechnol. 3 (2008) 563.

[7] D. Li, M.B. Muller, S. Gilje, R.B. Kaner, GG Wallace, Nat. Nanotechnol. 3 (2008) 101.

[8] D. Chen, L. Tang, J. Li, Chem. Soc. Rev. 39 (2010) 3157.

[9] C. Liu, Z. Yu, D. Neff, A. Zhamu, B.Z. Jang, Nano Lett. 10 (2010) 4863.

[10] Q. Cheng, J. Tang, J. Ma, H. Zhang, N. Shinya, L.C. Qin, Phys. Chem. Chem. Phys. 13 (2011) 17615.

[11] J. Zhao, L. Liu, F. Li, Graphene Oxide: Physics and Applications. 2015, Berlin: Springer.

[12] L. Mao, K. Zhang, H.S. On Chan, J. Wu, J. Mater. Chem. 22 (2012) 80.

[13] Z.J. Fan, Q.K. Zhao, T.Y. Li, J. Yan, Y.M. Ren, J. Feng, T. Wei, Carbon 50 (2012) 1699.

[14] Y. Zhu, S. Murali, M.D. Stoller, K.J. Ganesh, W. Cai, P.J. Ferreira, A. Pirkle, R.M. Wallace, K.A. Cychosz, M. Thommes, D. Su, E.A. Stach, R.S. Ruoff, Science 332 (2011) 1537. 
[15] J. Bai, X. Zhong, S. Jiang, Y. Huang, X. Duan, Nat. Nanotechnol. 5 (2010) 190.

[16] G Ning, Z. Fan, G Wang, J. Gao, W. Qian, F. Wei, Chem. Commun. 47 (2011) 5976.

[17] J.S. Bunch, S.S. Verbridge, J.S. Alden, A.M. van der Zande, J.M. Parpia, H.G Craighead, P.L. McEuen, Nano Lett. 8 (2008) 2458.

[18] B.Z. Jang, C. Liu, D. Neff, Z. Yu, M.C. Wang, W. Xiong, A. Zhamu, Nano Lett. 11 (2011) 3785.

[19] X. Zhao, C.M. Hayner, M.C. Kung, H.H. Kung, ACS Nano 5 (2011) 8739.

[20] T.G Pedersen, C. Flindt, J. Pedersen, N.A. Mortensen, A.-P. Jauho, K. Pedersen, Phys. Rev. Lett 100 (2008) 136804.

[21] G Luo, L. Liu, J. Zhang, G Li, B. Wang, J. Zhao, ACS Applied Materials \& Interfaces 5 (2013) 11184.

[22] H.Y. He, Y. Zhang, B.C. Pan, J. Appl. Phys. 107 (2010) 114322.

[23] F. Ouyang, S. Peng, Z. Liu, Z. Liu, ACS Nano 5 (2011) 4023.

[24] H. Jippo, M. Ohfuchi, C. Kaneta, Phys. Rev. B 84 (2011) 075467.

[25] Ç.Ö. Girit, J.C. Meyer, R. Erni, M.D. Rossell, C. Kisielowski, L. Yang, C.-H. Park, M.F. Crommie, M.L. Cohen, S.G Louie, A. Zettl, Science 323 (2009) 1705.

[26] M. Bieri, M. Treier, J. Cai, K. Ait-Mansour, P. Ruffieux, O. Groning, P. Groning, M. Kastler, R. Rieger, X. Feng, K. Mullen, R. Fasel, Chem. Commun. 45 (2009) 6919.

[27] Z. Liumin, K. Konishi, K. Yoh, Jap. J. Appl. Phys. 50 (2011) 06GE14.

[28] P. Giannozzi, S. Baroni, N. Bonini, M. Calandra, C. Roberto, C. Carlo, C. Davide, L.C. Guido, C. Matteo, D. Ismaila, C. Andrea Dal, G Stefano de, F. Stefano, F. Guido, G Ralph, G Uwe, G Christos, K. Anton, L. Michele, M.-S. Layla, M. Nicola, M. Francesco, 
M. Riccardo, P. Stefano, P. Alfredo, P. Lorenzo, S. Carlo, S. Sandro, S. Gabriele, P.S. Ari, S. Alexander, U. Paolo, M.W. Renata, J. Phys.: Condens. Mat. 21 (2009) 395502.

[29] J.P. Perdew, K. Burke, M. Ernzerhof, Phys. Rev. Lett. 77 (1996) 3865.

[30] D.R. Hamann, Schl, uuml, M. ter, C. Chiang, Phys. Rev. Lett. 43 (1979) 1494.

[31] H.J. Monkhorst,J.D. Pack, Phys. Rev. B 13 (1976) 5188.

[32] M. Methfessel,A.T. Paxton, Phys. Rev. B 40 (1989) 3616.

[33] P. Koskinen, S. Malola, H. Häkkinen, Phys. Rev. Lett. 101 (2008) 115502.

[34] C. Berger, Z. Song, X. Li, X. Wu, N. Brown, C. Naud, D. Mayou, T. Li, J. Hass, A.N. Marchenkov, E.H. Conrad, P.N. First, W.A. de Heer, Science 312 (2006) 1191.

[35] K.I. Bolotin, K.J. Sikes, Z. Jiang, M. Klima, G Fudenberg, J. Hone, P. Kim, H.L. Stormer, Solid State Commun. 146 (2008) 351.

[36] C.-H. Park, L. Yang, Y.-W. Son, M.L. Cohen, S.G Louie, Nat. Phys. 4 (2008) 213.

[37] C.-H. Park, L. Yang, Y.-W. Son, M.L. Cohen, S.G Louie, Phys. Rev. Lett. 101 (2008) 126804.

[38] A. Zhang, H.F. Teoh, Z. Dai, Y.P. Feng, C. Zhang, Appl. Phys. Lett. 98 (2011) 023105.

[39] S. Kondrat, C.R. Perez, V. Presser, Y. Gogotsi, A.A. Kornyshev, Energy Environ. Sci. 5 (2012) 6474.

[40] C. Largeot, C. Portet, J. Chmiola, P.-L. Taberna, Y. Gogotsi, P. Simon, J. Am. Chem. Soc. $130(2008) 2730$.

[41] S. Kondrat, N. Georgi, M.V. Fedorov, A.A. Kornyshev, Phys. Chem. Chem. Phys. 13 (2011) 11359.

[42] E. Raymundo-Piňero, K. Kierzek, J. Machnikowski, F. Béguin, Carbon 44 (2006) 2498. 
[43] J. Chmiola, C. Largeot, P.-L. Taberna, P. Simon, Y. Gogotsi, Angew. Chem. Int. Ed. 47 (2008) 3392.

[44] J. Chmiola, G. Yushin, R. Dash, Y. Gogotsi, J. Power Sources 158 (2006) 765. 
Graphical Abstract

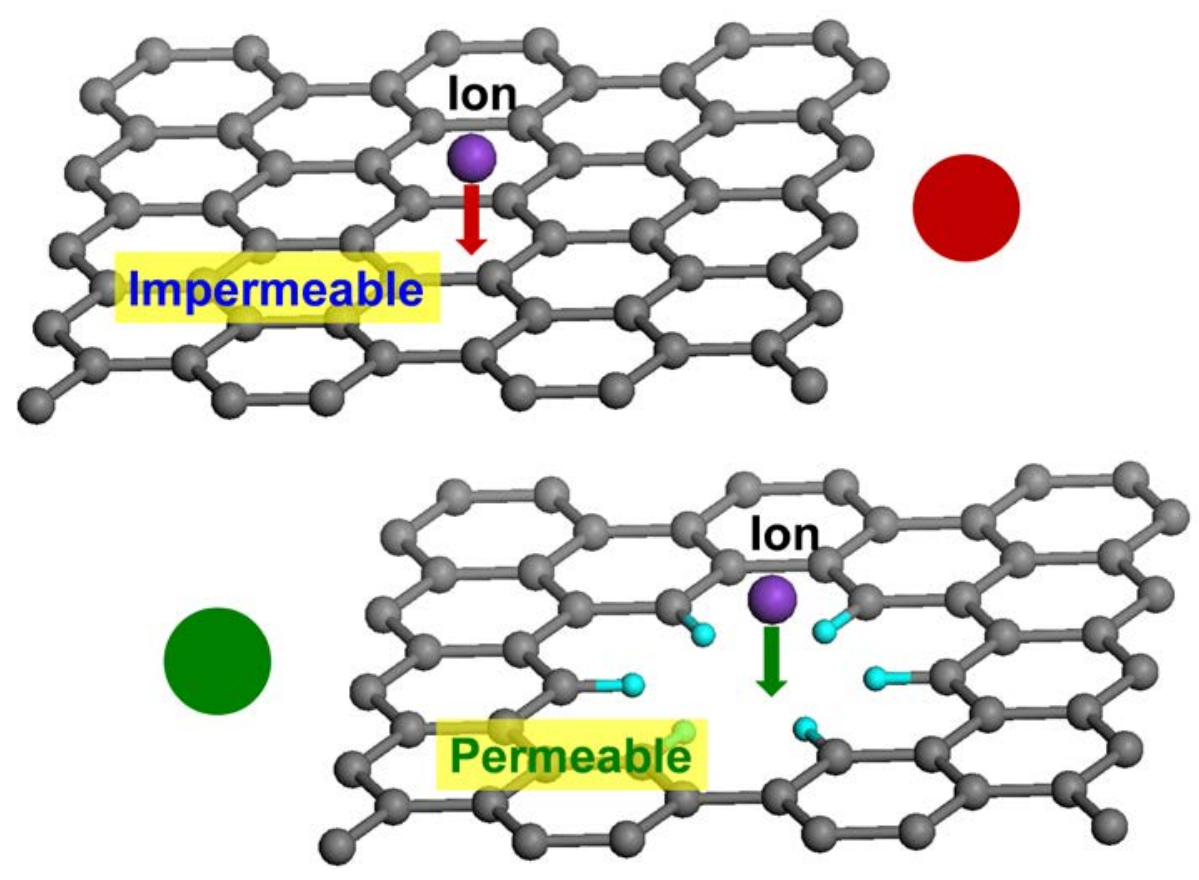

Compared with perfect graphene, porous graphene antidot lattices facilitate the diffusion of ions in passing through the graphene layers, which should be promising electrode materials for supercapacitors. 
(a)

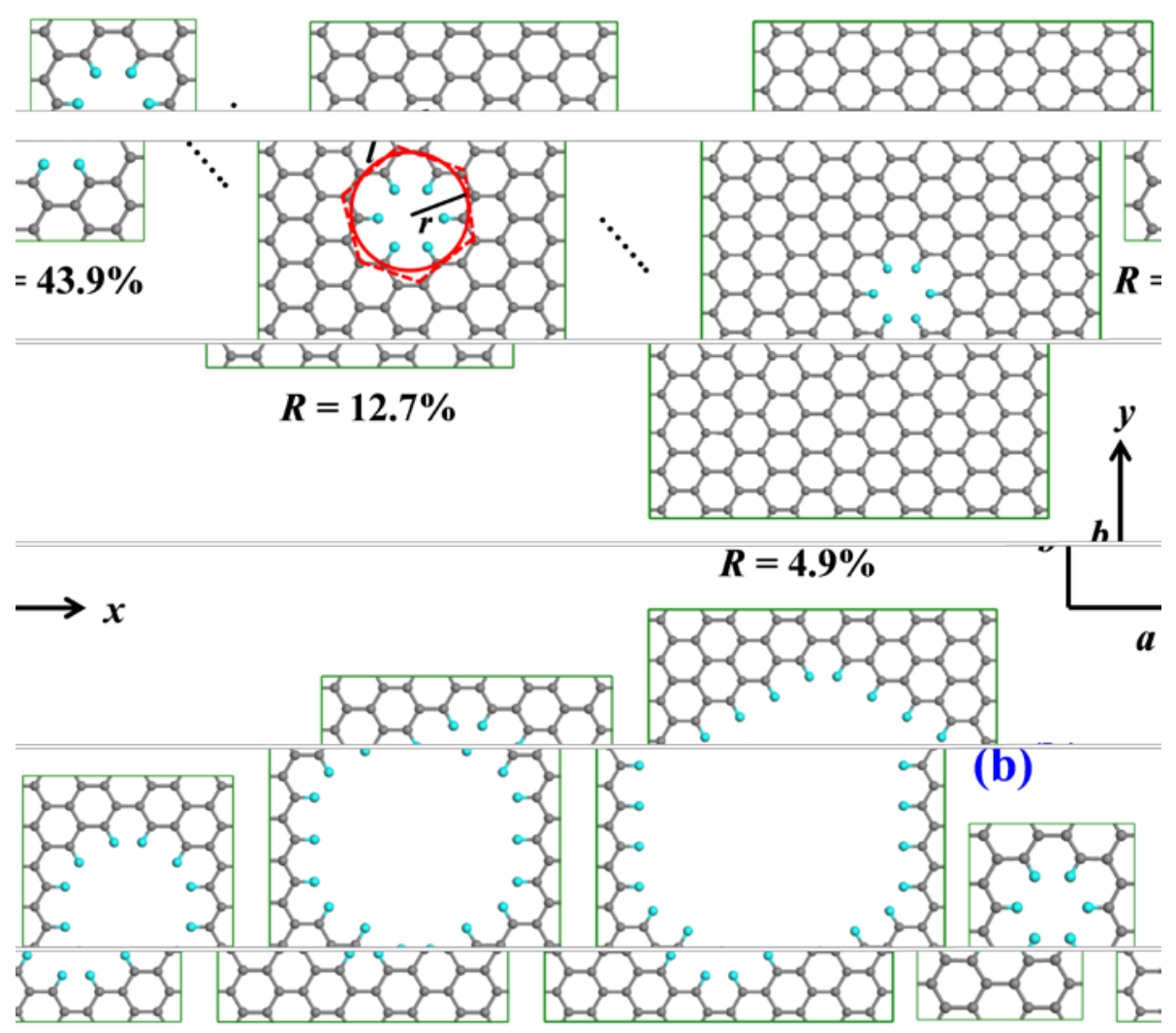

Fig. 1. (Color online) Structural models for the graphene antidot lattices with (a) different hole densities and (b) different hole sizes. The gray balls are $\mathrm{C}$ atoms and the light blue balls are $\mathrm{H}$ atoms. 


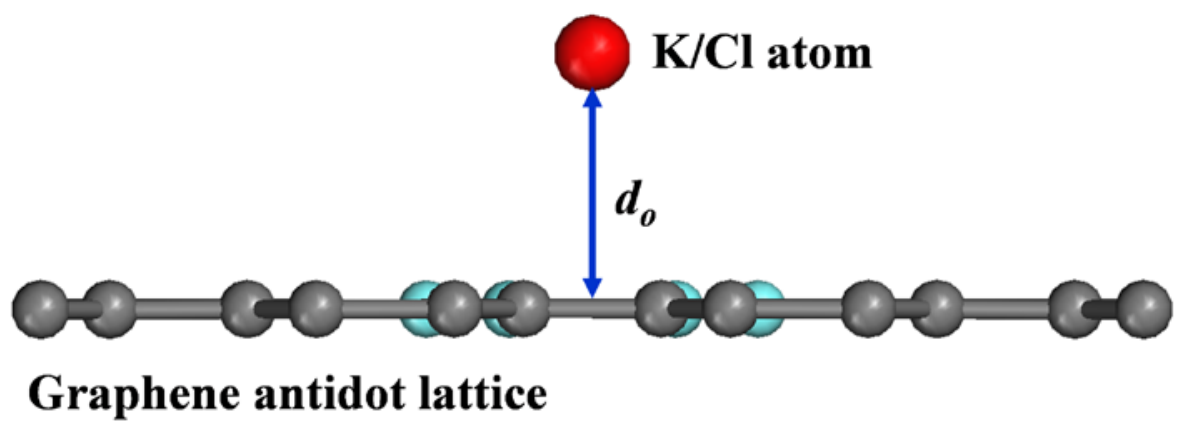

Fig. 2. (Color online) Schematic to show $\mathrm{K} / \mathrm{Cl}$ atom placed on graphene antidot lattice where $d_{o}$ is the optimal distance. The red ball is $\mathrm{K} / \mathrm{Cl}$ atom, the gray balls are $\mathrm{C}$ atoms and the light blue balls are $\mathrm{H}$ atoms. 


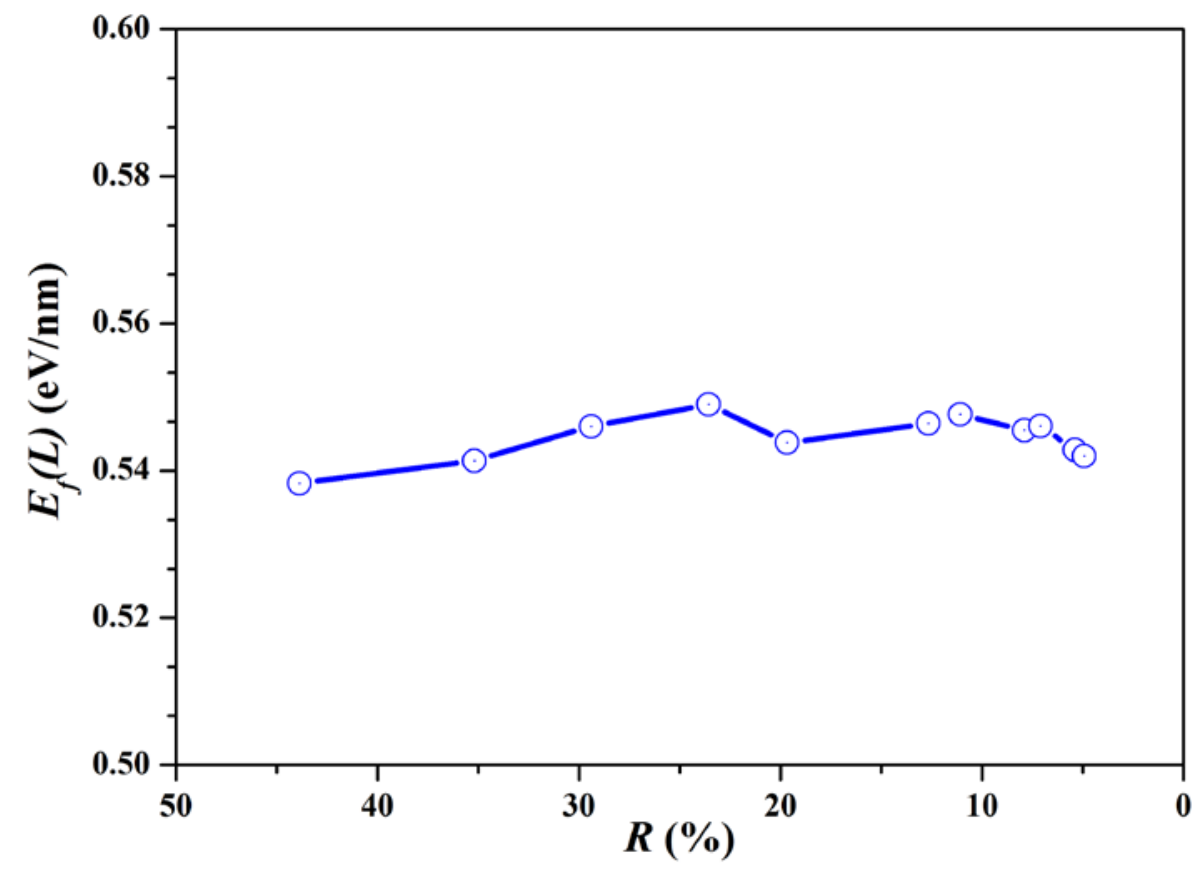

Fig. 3. (Color online) $E_{\text {form }}(L)$ as a function of $R$ for the graphene antidot lattices. 

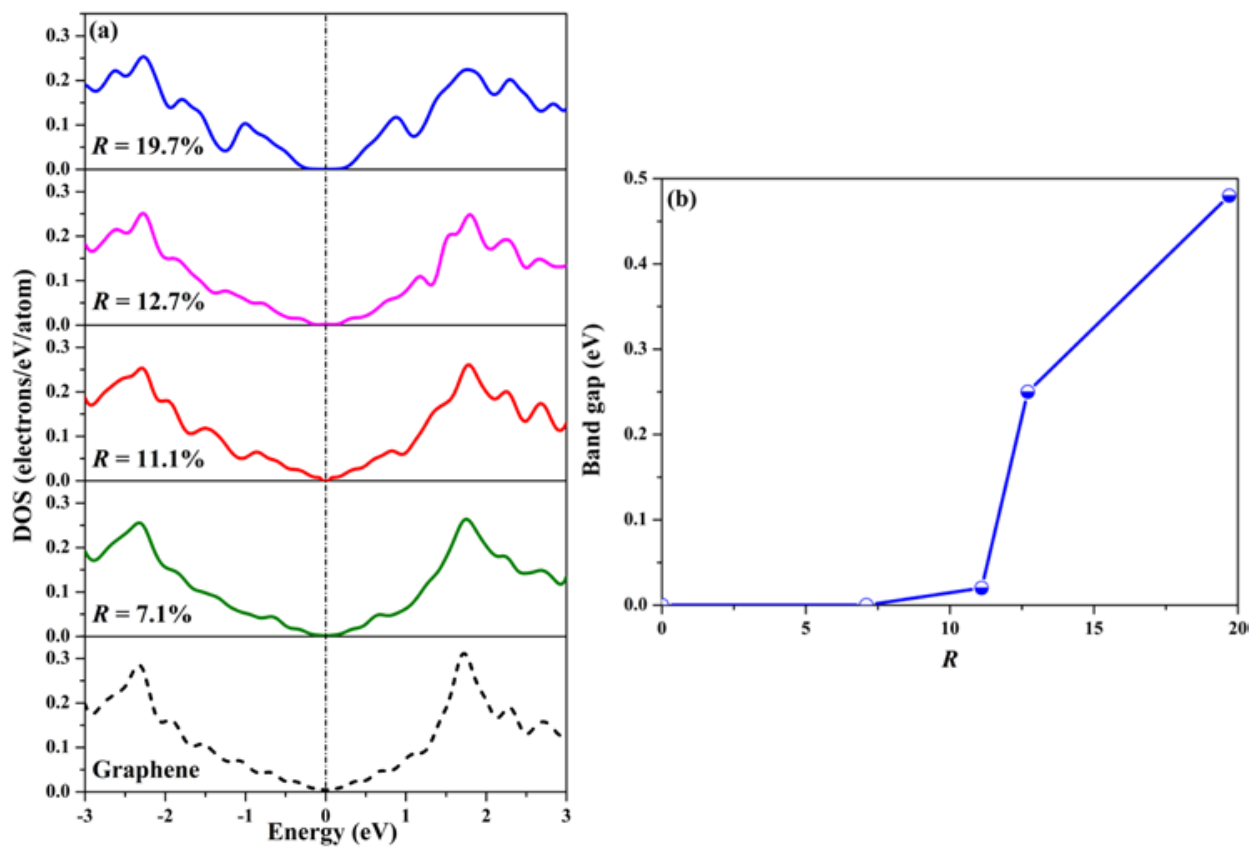

Fig. 4. (Color online) The electron DOS (a) and band gaps (b) of the graphene antidot lattices with different hole densities. The DOS of the perfect graphene is shown for reference (dashed line in the lower plot). The Fermi energy as dotted vertical line is set to be zero. The smearing width is taken to be 0.005 Ry. 


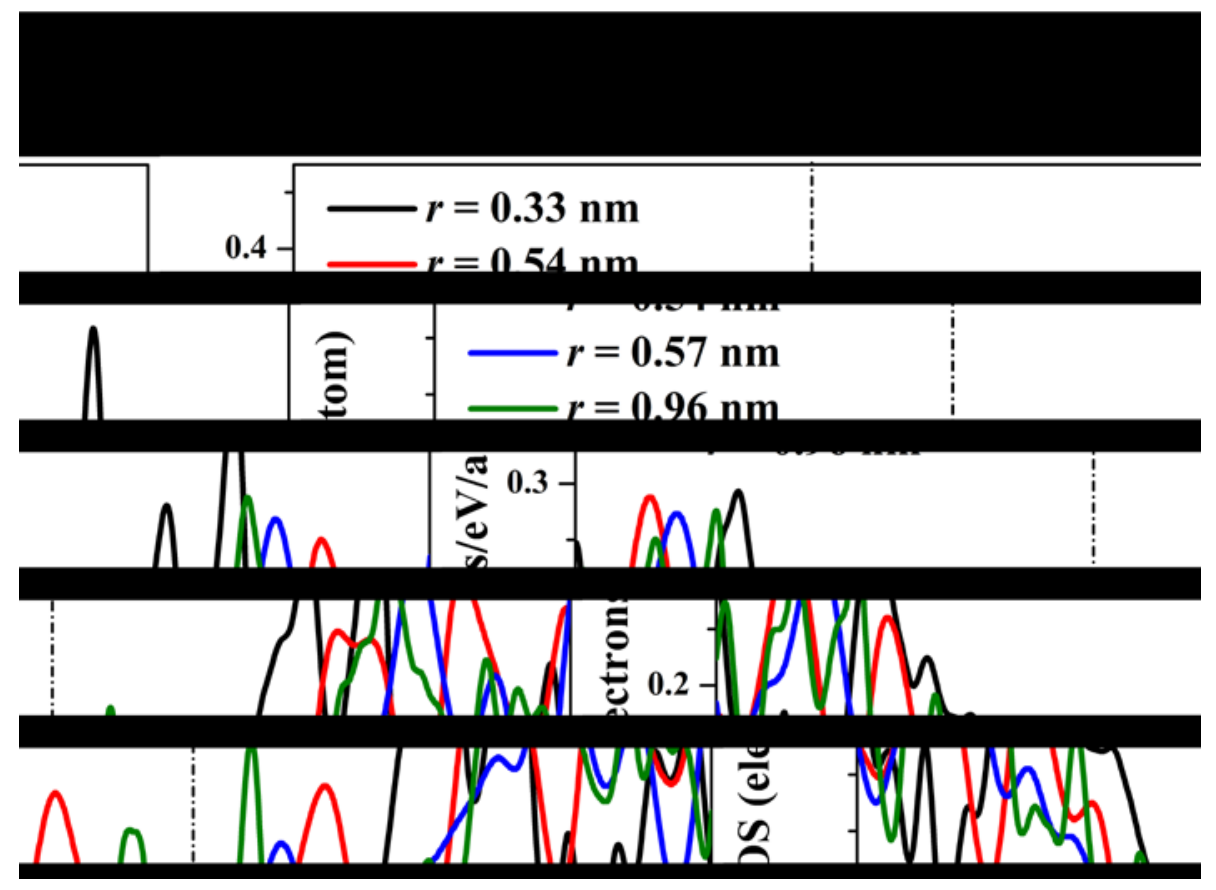

Fig. 5. (Color online) Electron DOS of the graphene antidot lattices with different hole sizes.

The Fermi energy as dotted vertical line is set to be zero. The smearing width was taken to be 0.005 Ry. 
Table 1

Formation energy per edge length $E_{\text {form }}(L)$ for holes with different hole size $r$ and edge length $L$.

\begin{tabular}{ccc}
\hline \hline$r(\mathrm{~nm})$ & $L(\mathrm{~nm})$ & $E_{F}^{L}(\mathrm{eV} / \mathrm{nm})$ \\
\hline 0.33 & 2.28 & 0.54 \\
0.54 & 3.72 & 0.53 \\
0.75 & 5.18 & 0.54 \\
0.96 & 6.67 & 0.57 \\
$\infty$ & $\infty$ & $0.60[33]$ \\
\hline \hline
\end{tabular}


Table 2

The maximum diffusion barrier $(\mathrm{eV})$ for $\mathrm{K}$ and $\mathrm{Cl}$ atoms going through the graphene or graphene antidot lattices with different hole sizes, respectively.

\begin{tabular}{ccccc}
\hline \hline & graphene & \multicolumn{3}{c}{ Graphene antidot lattice } \\
\cline { 2 - 4 } $\mathrm{K}$ & - & $r=0.33 \mathrm{~nm}$ & $r=0.54 \mathrm{~nm}$ & $r=0.75 \mathrm{~nm}$ \\
\hline $\mathrm{Cl}$ & 73.98 & 3.52 & 0.05 & 0.05 \\
\hline \hline
\end{tabular}

